Military Technical College Kobry El-Kobbah, Cairo, Egypt

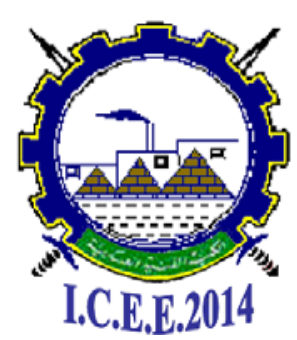

$7^{\text {th }}$ International Conference on

Chemical \& Environmental Engineering

27 - 29 May, 2014.

\title{
CEEB-1
}

\section{Studying the kinetics and mechanism of tartrazine removal by activated carbon under the influence of shaking speed using Diffusion-Binding model}

\author{
H. I. Albroomi**, Ahmad Baraka*, M. A. Elsayed*, M. Kassem*
}

\begin{abstract}
The kinetic and mechanistic study of the removal rate of tartarzine by adsorption onto activated carbon under the influence of shaking speed is presented in this work. Diffusion binding model (DB-model) and pseudo second order model (PSO-model) were applied for suggesting the dependency of surface-reaction (solute or active site) and to give the main outputs; adsorption rate constants and capacities. According to error evaluation of both models, it was found that DB-model is more representative for tartarzine activated carbon adsorption system than PSO-model and it is suggested that surface-reaction kinetic is dependent on tartrazine concentration not on active sites of adsorbent. In addition, DB-model showed that adsorption rate constants and capacities increase with shaking speed which agrees with the fact of enhancing some diffusion steps because of shaking. DB-model, as a diffusion model as well, was applied to investigate diffusion mechanistic and it was concluded that for static condition (shaking speed $=0 \mathrm{rpm}$ ), adsorption passes two stages, the first is controlled by bulk-diffusion and film-diffusion and the second is controlled by intraparticle-diffusion. For shaking speeds $25 \mathrm{rpm}$ and $50 \mathrm{rpm}$, bulk-diffusion and filmdiffusion almost have no contribution in controlling overall rate and consequently intraparticle-diffusion is the controlling step. For shaking speeds from 75 to $125 \mathrm{rpm}$, intraparticle-diffusion is suggested to have strong contribution in controlling adsorption process.
\end{abstract}

\section{Keywords:}

Kinetics, adsorption, diffusion, DB-model, tartrazine, activated carbon.

** Oman Armed Forces

* Egyptian Armed Forces 


\section{Introduction}

Adsorption process is employed extensively for contaminant separation in environmental engineering. Adsorption becomes effective by using porous adsorbents because of remarkable high surface area. Contaminant adsorption onto porous adsorbent is always accompanied by diffusion mechanisms which have a significant effect on adsorption rate [1].

Conducting investigation to determine the effectiveness of any adsorption system requires kinetic modeling to determine capacity and rate constant and in addition to understand the mechanism of the adsorption process. There are many kinetic models which are successful in predicting batch adsorption behavior. Kinetic modeling can be performed according to surface-reaction models and diffusion models. Surface-reaction models concern the nature of binding. Diffusion models investigate the mechanistic steps of adsorption process. In the absence of agitation/stirring/shaking, the removal rate of contaminant is dependent on the rate of external mass transfer and intra-particle transport [2]. External mass transfer in many cases is faster than intra-particle transport, thus the rate of adsorption in porous adsorbents is generally controlled by intra-particle diffusion within the pores network. In the presence of effective agitation, external mass transfer enhances and intraparticle-diffusion is considered to control adsorption rate [3,4]. Furthermore, intraparticle-diffusion may occur by several different rates depending on pore size distribution [5,6]. Diffusion behavior may therefore be determined by modeling the experimental uptake points to proper applied kinetic model.

For comprehensive study, an accustomed practice in many adsorption works is to apply the two types of modeling; surface-reaction and diffusion. The present work introduces the recently developed DB-model to determine adsorption rate behavior of tartrazine onto activated carbon (T/AC) under the influence of different shaking speeds (SH-S) according to two aspects; surface-reaction and diffusion. DB-model surface-reaction modeling indicates that DB-model is better representing T/AC adsorption system than PSO-model. Diffusion modeling by DB-model gives important information about diffusion mechanistic of T/AC adsorption system.

\section{Kinetic models}

\subsection{DB-model}

DB-model has been recently presented and its main postulations are the dependency of adsorption on the solute concentration and the effectiveness of diffusion during adsorption process. DB-model is applied according to its linear form [7]:

$$
\frac{C_{\mathrm{f}} C_{\mathrm{e}}}{C_{\mathrm{i}}} \ln C_{\mathrm{t}}-C_{\mathrm{t}}=S t^{n}-D, \quad \text { and slope, } S=k_{\mathrm{DB}} / n
$$

where $C_{\mathrm{i}}$ : initial concentration, $C_{\mathrm{f}}$ : final concentration , $C_{\mathrm{e}}$ : equilibration concentration, and $C_{\mathrm{t}}$ : concentration at any time $t$ and where $S, n, k_{\mathrm{DB}}$ and $D$ are equation-slope, time-power ( $n<$ 1), rate constant and integration constant respectively. The constant of equation (1) $D$ can be calculated according to the following expression [7]:

$$
D=\frac{C_{\mathrm{f}} C_{\mathrm{e}}}{C_{\mathrm{i}}} \ln C_{\mathrm{i}}-C_{\mathrm{i}}
$$


Plotting of $\ln \left[\left(C_{\mathrm{f}} \mathrm{C}_{\mathrm{e}} / C_{\mathrm{i}}\right) \ln C_{\mathrm{t}}-C_{\mathrm{t}}\right]$ against $t^{\mathrm{n}}$ should produce a line from which the adsorption rate constant $\left(k_{\mathrm{DB}}\right)$ and estimated adsorption capacity of DB-model $\left(q_{\mathrm{DB}}\right)$ can be calculated according to the following two expressions respectively [7]:

$$
\begin{array}{ll}
k_{\mathrm{DB}}=S \times n & \left(\mathrm{mg} \cdot \mathrm{l}^{-1} \cdot \mathrm{min}^{-n}\right) \\
q_{\mathrm{DB}}=S \times t^{n} \times V / m & \left(\mathrm{mg} \cdot \mathrm{g}^{-1}\right)
\end{array}
$$

where $V$ is the solution volume and $m$ is the used adsorbent mass. In this work; $C, V, m$ and $t$ have the following units respectively; ppm, l, g, and minute.

Applying eqn. (1) on system's kinetic data points should give a linear plot of $\left(\left(C_{\mathrm{f}} C_{\mathrm{e}} / C_{\mathrm{i}}\right) \ln C_{\mathrm{t}}\right.$ $\left.-C_{t}\right)$ against $t^{n}$. The linearity is achieved under the condition of properly selecting the value of the power $n$ by using a data sheet program. The proper selection of $n$-value should give the highest determination coefficient, $R^{2}$, of regressed line and in the same time the programcalculated value of the constant $D$ should be equal or very close to $\left(\left(C_{\mathrm{f}} C_{\mathrm{e}} / C_{\mathrm{i}}\right) \ln C_{\mathrm{i}}-C_{\mathrm{i}}\right)$ according to eqn. (2).

\subsection{PSO-model}

PSO-model is widely applied to describe the adsorption kinetics of many different adsorption systems. Its major proposals are dependency of adsorption rate on vacant adsorbent-sites and the chemi-sorption nature of the adsorption processes. PSO-model has the following known linear expression [8]:

$$
\frac{t}{q_{\mathrm{t}}}=\frac{1}{k_{\mathrm{PSO}} q_{\mathrm{PSO}}^{2}}+\frac{t}{q_{\mathrm{PSO}}}
$$

where $q_{\text {Pso }}$ and $q_{\mathrm{t}}$ (mg.g $\mathrm{g}^{-1}$ ) are the amounts of contaminant removed by adsorbent at equilibrium and at time $t$, respectively and $k_{\text {PSO }}\left(\mathrm{g} \cdot \mathrm{mg}^{-1} \cdot \mathrm{min}^{-1}\right)$ is the adsorption rate constant according to PSO-model.

\subsection{Error determination}

To evaluate applied kinetic models for selecting the proper one to represent adsorption system under study, determination coefficient $\left(R^{2}\right)$ of linear regression was considered. In addition relative error in estimating adsorption capacity $(R E \%)$ was also considered and was calculated according to the followinfg expression [9]:

$$
R E \%=100 \times\left(q_{\text {exp }}-q_{\text {model }}\right) / q_{\exp }
$$

\section{Experimental}

\subsection{Tartrazine solution and Activated carbon}

Tartrazine (molecular formula: $\mathrm{C}_{16} \mathrm{H}_{9} \mathrm{~N}_{4} \mathrm{Na}_{3} \mathrm{O}_{9} \mathrm{~S}_{2}$ ) was supplied by Morgan Chemical Company and were used without further purification. Tartrazine (100 mg) was applied into deionized water to prepare $100 \mathrm{ppm}$ working solution and $\mathrm{pH}$ was adjusted to be 6.8. Activated carbon was also supplied by Morgan Chemical Company and has been used as adsorbent without further chemical treatment. A measured quantity of activated carbon was immersed in hot water for three hours to remove some potential contamination. The washed 
activated carbon was filtered and oven dried at $60^{\circ} \mathrm{C}$ for 2 hours and then heated to $200{ }^{\circ} \mathrm{C}$ for 3 hours with flow of $\mathrm{N}_{2}$ to hinder oxidation by air.

\subsection{Batch adsorption experiments}

SH-S is the factor to study in this work (0, 25, 50, 75, 100, 125 rpm). For each experiment, tartarzine solution $(100 \mathrm{ml})$ was treated by active carbon $(0.5 \mathrm{~g})$ with applied temperature $\left(25^{\circ} \mathrm{C}\right)$ at selected SH-S using Companion SI 300R shaker. Periodically, every 10 minutes, 3 $\mathrm{ml}$ was withdrawn from solution flask for absorbance measurement (at $\lambda_{\max }=425$ ) using Agilant UV-Vis Cary 60PC scan spectrophotometer using glass cell of $1 \mathrm{~cm}$ width.

\section{Results and discussion}

\subsection{General comments on adsorption behavior}

Fig. 1 shows the change of removal percentage $(R \%)$ of tartrazine with time. Adsorptiveremoval of is gradual and smooth for all applied SH-S and as SH-S increases removal percentage increases. This is an important phenomenon points to the remarkable effect of shaking on the adsorption effectiveness and it can be related to the enhancement of diffusion rates due to influencing the distribution of solute in bulk solution and decrease the resistance of the boundary film $[2,3]$.

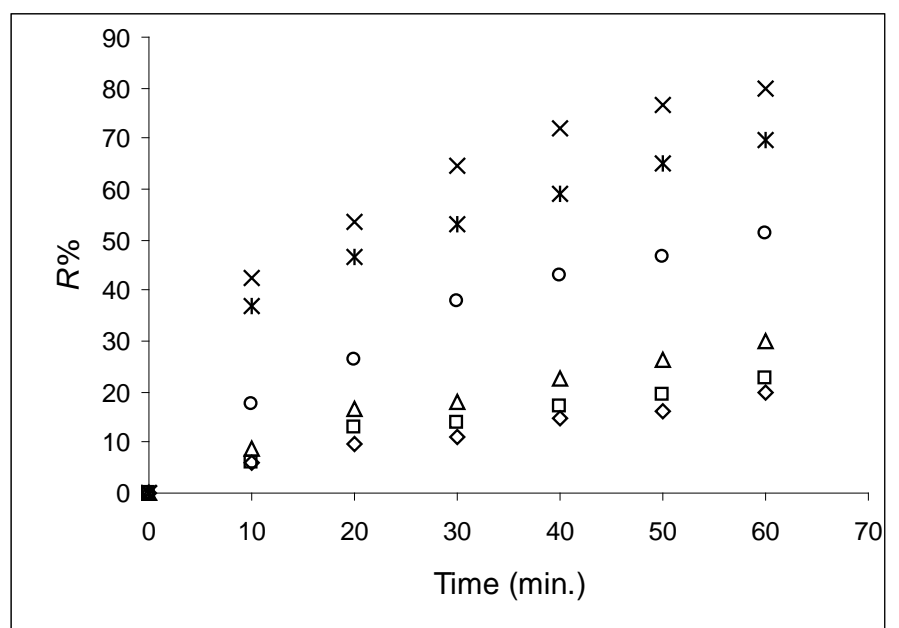

Fig. 1. Change of removal percentage of tartrazine with time for SH-S: $0(\diamond), 25(\square), 50(\Delta), 75(\mathrm{o}), 100(*), 125 \mathrm{rpm}(\times)$

\subsection{Surface-reaction modeling: Application of DB-model and PSO-model}

Fig. 2 shows the kinetic plots of T/AC adsorption system by modeling using DB-model and PSO-model. The outputs of these plots were calculated and are given in Table 1. 

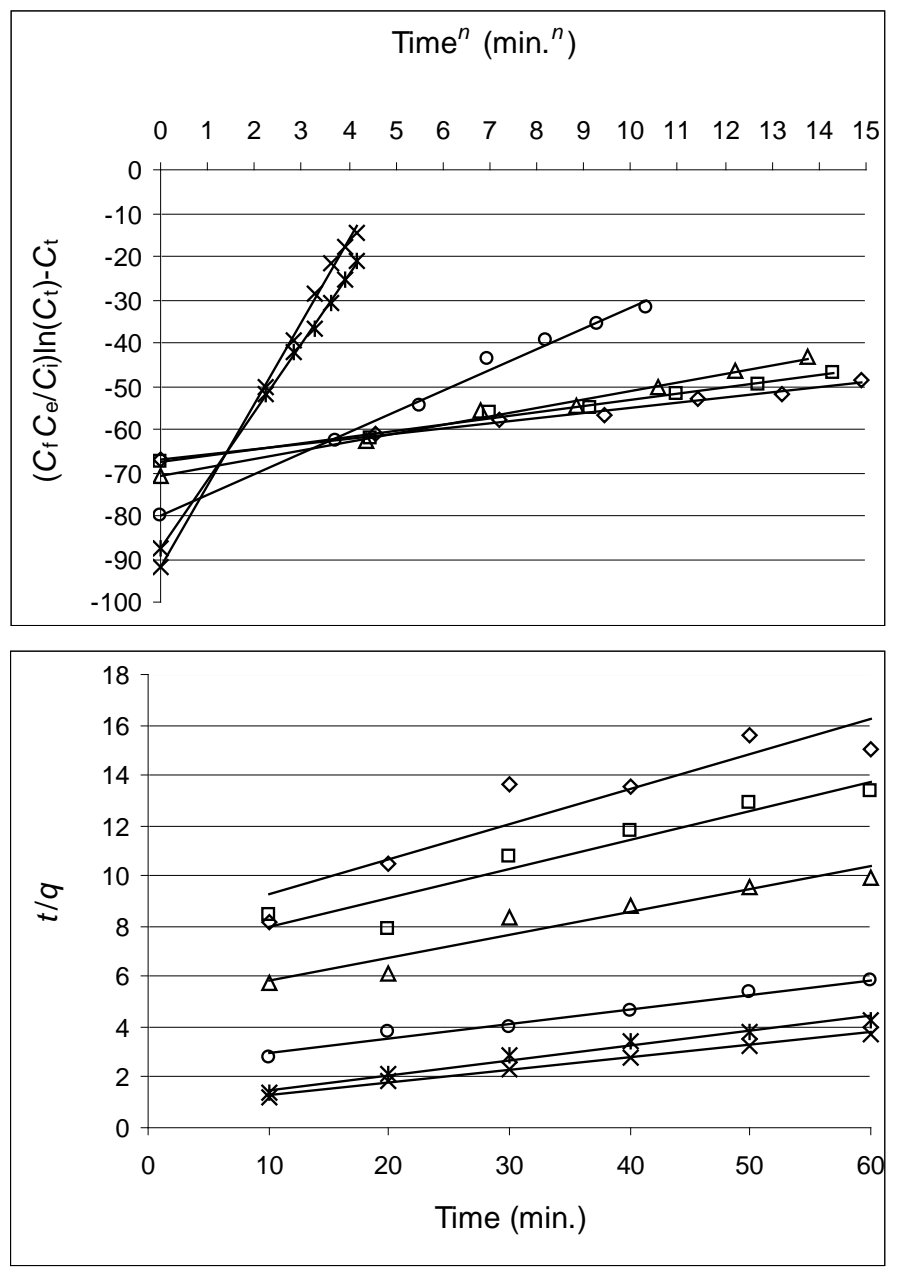

Fig. 2. Plots of DB-model (A)and PSO-model (B) for SH-S: $0(\diamond), 25(\square), 50(\Delta), 75(0), 100(*), 125 \mathrm{rpm}(\times)$

Table 1: Outputs of modeling by DB-model and PSO-model

\begin{tabular}{|c|c|c|c|c|c|c|c|c|c|c|}
\hline \multirow{3}{*}{ SH-S } & EXP. & \multicolumn{6}{|c|}{ DB-model } & \multicolumn{3}{|c|}{ PSO-model } \\
\cline { 2 - 11 } & $\boldsymbol{q}_{\mathbf{E X P}}$ & $\boldsymbol{n}$ & $\boldsymbol{k}_{\text {DB }}$ & $\boldsymbol{q}_{\mathbf{D B}}$ & $\boldsymbol{R E} \%$ & $\boldsymbol{R}^{2}$ & $\begin{array}{c}\boldsymbol{k}_{\text {PSO }} \\
\left(\times 10^{-3}\right)\end{array}$ & $\boldsymbol{q}_{\text {PSO }}$ & $\boldsymbol{R E} \%$ & $\boldsymbol{R}^{\mathbf{2}}$ \\
\hline 0 & 4.00 & 0.66 & 0.7763 & 3.51 & 12.25 & 0.9891 & 2.5197 & 7.12 & 78.00 & 0.8561 \\
\hline 25 & 4.48 & 0.65 & 0.9324 & 4.11 & 8.26 & 0.9873 & 1.9780 & 8.62 & 92.41 & 0.9075 \\
\hline 50 & 6.02 & 0.64 & 1.2811 & 5.50 & 8.64 & 0.9922 & 1.6904 & 10.98 & 82.39 & 0.9231 \\
\hline 75 & 10.24 & 0.57 & 2.7322 & 9.89 & 3.42 & 0.9927 & 1.4804 & 16.98 & 65.82 & 0.9829 \\
\hline 100 & 13.96 & 0.35 & 5.5076 & 13.19 & 5.52 & 0.9993 & 3.6425 & 17.15 & 22.85 & 0.9856 \\
\hline 125 & 15.98 & 0.35 & 6.5646 & 15.72 & 1.63 & 0.9981 & 3.2797 & 19.92 & 24.66 & 0.9956 \\
\hline
\end{tabular}

From Table 1, referring to determination coefficient values $\left(R^{2}\right)$ of both models, it can be concluded that DB-model is better to represent adsorption kinetics of T/AC adsorption system than PSO-model. In addition, referring to relative error values of adsorption capacity of both models $(R E \%)$, the estimated adsorption capacities by DB-model are also more accurate than those estimated by PSO-model. Accordingly, it is suggested that surface-interaction of T/AC adsorption system is dependent on tartrazine concentration not on vacant active sites of adsorbent as PSO-model may indicate [7,8]. This suggestion can be supported by recalling that the product of sample volume $(V)$ and concentration $(C)$ is higher than the product of adsorbent mass $(m)$ and sorption capacity $(q)$ for all applied SH-H settings. 
According to DB-model, $n$-values are less than 1 and this indicates remarked contribution of diffusion mechanisms in the adsorption process for all applied SH-S [7]. Furthermore, from table, as SH-S increases from 0 to $125 \mathrm{rpm}, n$-value decreases from 0.66 to 0.35 which indicates increase of contribution of diffusion mechanisms in controlling overall rate of adsorption. This can be understood from the fact that adsorption amount increases with SH-S and this means more accommodation of tartrazine into deep carbon pores which requires more intraparticle diffusion. The rate constant of DB-model increases regularly from 0.7763 to 6.5646 with SH-S. This significant increase suggests that rate constant is not only dependent on surface-reaction but also depends on enhanced diffusion.

\subsection{Diffusion modeling: Application of DB-model}

DB-model was empirically developed considering the assumption that diffusion has contribution in adsorption process. This was presented by considering proportionality with $t^{n}$. Hence; this model is able to recognize diffusion mechanism as presented in the previous section. However, the model can further give some more detailed information about diffusion mechanism when applied considering segmentation of adsorption period into observed stages. Fig. 3 shows DB-model plots according to this application by dividing adsorption whole period into two stages, solid line represents first stage and dashed line represents second stage. The outputs of these plots are summarized in Table 2.

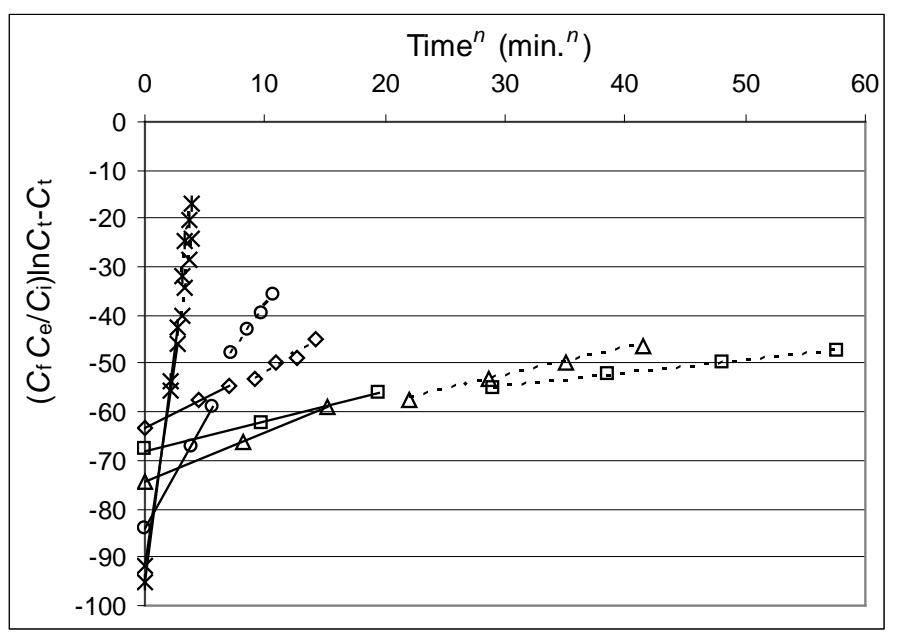

Fig. 3. Plots of DB-model considering segmentation for SH-S: $0(\diamond), 25(\square), 50(\Delta), 75(\mathrm{o}), 100(*), 125 \mathrm{rpm}(\times)$

For adsorption processes on porous adsorbents, diffusion mechanisms contribute effectively in the overall adsorption rate [10,11]. The steps that contribute in adsorption processes commonly considered as follows: (I) bulk-diffusion, (II) film-diffusion, (III) intraparticlediffusion and finally (IV) surface-reaction [10]. From Table 2, for SH-S= $0 \mathrm{rpm}$ adsorption amount is the lowest and rate constants of both stages are almost similar and $n=0.65$. This means that both external diffusion rate (bulk-diffusion and film-diffusion) and intraparticlediffusion rate are of the similar speed and slower than surface reaction step and consequently control the overall rate of adsorption [11]. For SH-S= $25 \mathrm{rpm}$ and $50 \mathrm{rpm}$, ₹n 1 which $\mathrm{s}$ indicate that external diffusion is of the same order of surface-reaction. however, rate constant of second stage is about half that of the first stage and consequently intraparticle-diffusion controls the rate during the second stage and this was determined from table 1 as $\mathrm{n} \approx 0.65$ and 0.64 [12]. For SH-S= $75 \mathrm{rpm}, \mathrm{n} \approx 0.58$ and rate constants of the two stages are almost similar 
and in addition rate constant of the second stage is considerably higher than corresponding rate constants of SH-S $<75 \mathrm{rpm}$. This indicates that intraparticle-diffusion of the second stage becomes more controlling. For SH-S $=100 \mathrm{rpm}$ and $125 \mathrm{rpm}, \mathrm{n} \approx 0.33$, rate constants of the two stages are almost similar and in addition rate constant of the second stage is considerably higher than corresponding rate constants of SH-S $<100 \mathrm{rpm}$ which indicates that intraparticle-diffusion of the second stage becomes more and more controlling [13].

Another important feature is that the second stage shares significantly in the amount adsorbed. Adsorbed amounts during second stage have a range from $27 \%$ to $54 \%$ of the total capacities which means that during second stage intraparticle-diffusion is responsible for controlling the overall rate of adsorption.

Table 2: Outputs of diffusion modeling by DB-model

\begin{tabular}{|c|c|c|c|c|c|c|c|c|cc|}
\hline \multirow{2}{*}{ SH-S } & EXP. & \multicolumn{10}{|c|}{ DB-model } \\
\cline { 2 - 12 } & $\boldsymbol{q}_{\text {EXP }}$ & $\boldsymbol{n}$ & $\boldsymbol{k}_{\text {DB-S1 }}$ & $\boldsymbol{k}_{\text {DB-S2 }}$ & $\boldsymbol{q}_{\text {DB-S1 }}$ & $\boldsymbol{q}_{\text {DB-S2 }}$ & $\boldsymbol{q}_{\text {DB }}$ & $\boldsymbol{R}_{\text {DB }} \boldsymbol{\%}$ & $\boldsymbol{R}_{\text {DB-S1 }}^{2}$ & $\boldsymbol{R}_{\text {DB-S2 }}^{2}$ \\
\hline 0 & 4.00 & 0.65 & 0.8078 & 0.9582 & 1.74 & 2.15 & 3.89 & 2.75 & 1.0000 & 0.9632 \\
\hline 25 & 4.48 & 0.99 & 0.5992 & 0.2650 & 2.35 & 2.04 & 4.39 & 2.01 & 0.9981 & 0.9978 \\
\hline 50 & 6.02 & 0.91 & 0.9174 & 0.5238 & 3.08 & 3.02 & 6.10 & 1.33 & 1.0000 & 0.9974 \\
\hline 75 & 10.24 & 0.58 & 2.5974 & 2.0018 & 5.09 & 3.50 & 8.59 & 16.11 & 1.0000 & 0.9977 \\
\hline 100 & 13.96 & 0.33 & 5.6054 & 6.8026 & 9.13 & 4.84 & 13.97 & 0.07 & 1.0000 & 0.9987 \\
\hline 125 & 15.98 & 0.33 & 6.4921 & 6.0707 & 10.57 & 4.32 & 14.89 & 6.82 & 1.0000 & 0.9853 \\
\hline
\end{tabular}

\section{Conclusion}

The kinetics and mechanism of T/AC adsorption system has been presented. From surfacereaction kinetic discussion, DB-model was found to be more representative than PSO-model suggesting that adsorption is dependent on tartrazine concentration. Adsorption capacity and rate constant increase regularly and significantly with SH-S indicating enhancement in diffusion because of shaking, however, diffusion becomes more controlling the overall rate due to more contribution of intraparticle-diffusion in adsorption process. Diffusion mechanistic discussion revealed that for low SH-S, both external diffusion rate and intraparticle-diffusion rate control the overall rate of adsorption. For mid SH-S (25-50 rpm), intraparticle-diffusion controls the overall rate of adsorption. For high SH-S (75-125 rpm), intraparticle-diffusion strongly controls the overall rate of adsorption. In general DB-model successfully represents T/AC adsorption system according for both surface-reaction and diffusion aspects.

\section{References}

[1] Kumar Djamel Belaid, Smaïl Kacha, Mostefa Kameche, Zoubir Derrche, Adsorption kinetics of some dyes onto granular activated carbon, Journal ofEnvironmental Chemical Engineering, 1 (2013) 496-503.

[2] Loubna Nouri, Ilhem Ghodbane, Oualid Hamdaoui, Mahdi Chiha, Batch sorption dynamics and equilibrium for the removal of cadmium ions from aqueous phase using wheat bran, Journal of Hazardous Materials, 149 (2007) 115-125

[3] B.K. Nandi, A. Goswami, M.K. Pukait, Adsorption characteristics of brilliant green dye on kaolin, Journal of Hazardous Materials, 161 (2009) 387-395.

[4] S.Figaro, J.P. Avril, F. Brouers, a. Ouensaga, S. Gaspard, Adsorption studios of molasse's wastewater on activated carbon: Modelling with a new fractal kinetic equation and evaluation of kinetic models, Journal of Hazardous Materials, 161 (2009) 649-656. 
[5] Augustine E. Ofomaja, Emmanuel I. Unuabonah, Adsorption kinetics of 4-nitrophenol noto cellulosic material, mansonia wood sawdust and multistage batch adsorption process optimization, Carbohydrate Polymers, 83 (2011) 1192-1200.

[6] Mohammad I. El-khaiary, Gihan F. Malash, Common data analysis errors in batch adsorption studies, Hydrometallurgy, 105 (2011) 314-320.

[7] Ahmad Baraka, A novel kinetic model for batch aqueous-solution /porous-solid adsorption systems, Desalination and Water Treatment, (2013) 1-14, DOI: 10.1080/19443994.2013.807479.

[8] Y.S. Ho, G. McKay, The kinetics of sorption of divalent metal ions onto sphagnum moss peat, Water Research, 34 (2000) 735-742.

[9] Saied Azizian, Rahimeh Naviri Fallah, A new empirical rate equation fro adsorption kinetics at solid/solution interface, Applied Surface Science, 256 (2010) 5153-5156.

[10] Wojciech Plazinski, Wladyslaw Rudzinski, Anita Plazinski, theoriticaal models of sorption kinetics including a surface reaction mechanism: A review, Advances in Colloid and Interface Science, 152 (2009) 2-13.

[11] Gihan F. Malash, Mohammed I. El-Khaiary, Piecewise linear regression: A statistical method for the analysis of experimental adsorption data by the intraparticle-diffusion models, Chemical Engineering Journal, 163 (2010) 256-263.

[12] Władysław Rudzinski, Wojciech Paziniski, Kinetics of metal ions adsorption at heterogeneous solid/solution interfaces: A theoretical treatment based on statistical rate theory, Journal of Colloid and Interface Science, 327 (2008) 36-43.

[13] Suhong Chen, Jian Zhang, Chenglu Zhang, Qinyan Yue, Yan Li, Chao Li, equilibrium and kinetics of mrthyl orange and methyl violet adsorption on activated carbon from Phragmites australis, Desalination 252 (2010) 149-156. 